\title{
26. COMMISSION DES ETOILES DOUBLES
}

\author{
President: M. R. G. Aitken, Associate Director, Lick Observatory, Mount \\ Hamilton, California, U.S.A.
}

Membres: MM. Bigourdan, Dawson, Espin, Fox, Gabba, Hertzsprung, Innes, Jackson, Luplau-Janssen, Olivier, Van Biesbroeck, Van den Bos.

The Chairman of the Commission on Double Stars sent out letters to all members of the Commission in July 1926, and in June 1927, presenting a number of propositions for their consideration and asking for additions, amendments and suggestions. Many of these related to the form and content of the Extension to Burnham's General Catalogue. Replies to both letters were received from all of the members and the present report is believed to embody the views of the entire Commission. Naturally, opinions differed on some points, but the general agreement was gratifying. On points of difference, the view of the majority has been adopted.

Double-star observers have been very active in the three years' period covered by this report: one large volume of measures and a great many shorter lists have been published. Two volumes call for specific mention: Vol. 5, No. I, Publications of the Yerkes Observatory, containing, in Part I, the measures by Professor G. van Biesbroeck of all of the $\mathrm{Hu}$ (Hussey) double stars discovered at the Lick Observatory and many of those discovered at La Plata, and in Part II, measures of many well-known binary systems with new orbits of nine of them. Volume 2, Publications of the Dearborn Observatory, which contains in Part I measures of all the double stars discovered with the I81-inch telescope by Burnham; in Part II, measures of a long list of miscellaneous pairs; and, in a final section, a short list of new pairs. The measures included in this volume were made in the period from I9I5 to I925. Reference should be made to the continued activity of Rev. T. E. H. Espin, and Mr Milburn, in their programme of discovery and measurement of fainter pairs, and also to the information received from the Pulkowo Observatory that since 1927 regular observations have been resumed with the I5-inch refractor.

The most important and most encouraging work of the three-year period, however, is that done at the Union Observatory in South Africa. The 28-inch telescope, so long delayed in its completion, was finally put into commission, about two years ago, and has since been in constant and successful use in carrying out Dr Innes's plan to make a systematic survey of the southern heavens for the discovery and measurement of close double stars. Dr Innes and the Chairman of this Commission have been in active correspondence for many years, and at the latter's suggestion, this survey, originally planned to begin at $-19^{\circ}$, the northern limit of the Cape Photographic Durchmusterung, is being extended to $-18^{\circ}$. This overlaps the Lick Observatory survey by $4^{\circ}$ in the 12 hours from $13^{\text {h }}$ to $I^{n}$ R.A., but the resurvey is worth while, for that zone is so low in the sky at Mount Hamilton that observations are usually difficult.

$\mathrm{Dr}$ W. H. van den Bos, who was seconded from the Leiden Observatory to the Union Observatory, greatly assisted Dr Innes in this survey, in which Mr W. S. Finsen has also taken part. 
All stars to $9 \cdot 0$ photographic magnitude are included in the survey, and, to make it more fully comparable with the northern survey (which includes the $9 \cdot 0$ B.D. vis. mag.), somewhat fainter stars of the later spectral classes are also being examined.

The new discoveries to the present date exceed $I_{500 .}$

It is understood that the Union Observatory is leaving a special zone of the southern sky to be examined by the members of the Lamont Expedition at Bloemfontein, which was organized by the late Professor W. J: Hussey. His tragic death, while en route to South Africa to set up the Lamont telescope brought sorrow to all his friends and a great loss to astronomy. It is gratifying to know that the work of the expedition, essentially as he had planned it, is to be carried out as a memorial to him.

In addition to the excellently planned programme of discovery and observation of double stars, Dr Innes and his colleagues have also published a new Preliminary Catalogue of Southern Double Stars, issuing it in parts including the pairs in a single hour of right ascension from $-\mathrm{I} 9^{\circ}$ to the south pole. Every effort has been made to give in the most condensed form possible all information that can be secured for each pair included. In all essentials the catalogue corresponds closely to the Extension to Burnham's General Catalogue which is in preparation at the Lick Observatory. The abbreviations for observers' names, the epochs adopted for the star places, and the general limits to be observed in deciding whether or not to include a given pair of stars, were all mutually agreed upon, to make the two catalogues as nearly comparable as possible. The fact that the Southern Catalogue begins at - $19^{\circ}$, while the Extension to Burnham's Catalogue goes as far south as $-30^{\circ}$ does not mean unnecessary duplication. The overlap will have several advantages; in particular, the advantage that, in general, northern observers, in planning their work, need consult only the Extension whereas southern observers, if they heed Innes's counsel, will confine their programmes to pairs in the Southern Catalogue.

It is desirable that, when the Southern Double Star Survey is completed, the Catalogue should be reissued in more permanent form.

We regret to report that Dr Innes was obliged to retire from the directorship of the Union Observatory on December 3I, I927, under the rules of the Union of South Africa for the retirement of government officials. It is to be hoped that the splendid work he has initiated and carried out so well will be continued by able successors. It would be nothing less than a catastrophe if it were to be discontinued.

The Extension to Burnham's General Catalogue, to which reference has already been made; is progressing very satisfactorily. In June 1926, Dr John C. Merriam; President of the Carnegie Institution, agreed to recommend the work for publication by the Institution as soon as the manuscript was ready. Preparations were at once made to close the card catalogue (so far as immediate publication was concerned) on January I, I927, and notice to this effect was sent to all double star observers. The Lick Observatory had given the Chairman the services of a half-time assistant for work on the catalogue as early as I925; a grant from the Research Fund of the University of California permitted the employment of another thoroughly competent assistant in May, I927. The result is that printer's copy at this date (January I5, I928) is ready for the stars to $9^{h}, R$.A. It is hoped that the entire manuscript may be ready to send to the printer early in I929.

Since this is an Extension to Burnham's Catalogue and not an entirely new 
work, the measures printed or referred to in the General Catalogue are not listed in it. Further, many of the fainter and wider pairs of the General Catalogue are omitted, the limit for inclusion being set by the formula $\log \rho=c-0.2 m$ where $m$ is the B.D. visual magnitude, and $c$ is $2 \cdot 8$, to make $\log \rho=I \cdot 0$ for $9 \cdot 0$ mag.

All pairs with angular distances falling within the limits set by this curve will be included in the Extension; other pairs will be included only when specific reasons exist for this inclusion, but, of course, the limit will not be followed too rigorously.

As in the Southern Double Star Catalogue, every effort is being made to present the data for each pair given in the Extension to Burnham's General Catalogue in the most compact form possible, and, while avoiding futile notes, to give, in addition to the measures, all available information as to orbital motion, spectral class, proper motion, parallax, and other particulars of astrophysical interest.

Orbit computation has received considerable attention during the past three years; improved orbits have been computed for many pairs, provisional orbits for a few others and new methods of orbit computation have been published by Dr Kurt Laves and by Dr van den Bos. The latter has also published a list of all stars for which orbits are available, giving the best orbit for each pair, and Dr Luplau-Janssen has published a complete catalogue of all orbits so far computed.

After this report of progress, it remains to suggest a few subjects that should receive the attention of the Double Star Commission at the Leiden Meeting. One of these is the question of suitable abbreviations to be recommended for the New Southern Double Star Catalogue and the Extension to Burnham's General Catalogue.

A second is the important matter of avoiding unnecessary duplication of measures of certain easy pairs and the total neglect of other pairs. This is a matter requiring very careful consideration, for while it is obvious that the end to be attained is most desirable, it is not easy to frame recommendations to secure that it will not interfere with the observer's initiative. This must be preserved. It may be noted that the General Card Catalogue of Double Star Measures is being kept to date, and that the Chairman of the Commission is at all times ready to supply data from it to any astronomer, and to furnish observers with lists of pairs that require observation.

Action should be taken to encourage the photographic measurement of pairs that can be separated on the photographic plate, and at the same time to investigate more carefully the nature and amount of the systematic errors inherent in such measures of close pairs. It has been suggested that a short list of pairs be compiled suited to the investigation of such errors and suited at the same time to the investigation of the personal errors of visual observers. Possibly a subcommittee could be named to prepare such a list.

ROBERT G. AITKEN

President of the Commission 